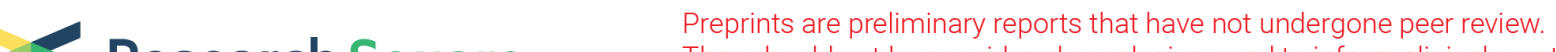 Research Square
or referenced by the media as validated information.
}

\section{Comparing the safety and efficacy of endovascular treatment versus bypass surgery for infrarenal aortic occlusion: a single-center experience}

\section{Jingjing Wang}

Chinese Academy of Medical Sciences \& Peking Union Medical College Hospital Jiang Shao

Chinese Academy of Medical Sciences \& Peking Union Medical College Hospital Chenyang Qiu

Chinese Academy of Medical Sciences \& Peking Union Medical College Hospital

\section{Lijing Fang}

Chinese Academy of Medical Sciences \& Peking Union Medical College Hospital

\section{Kang Li}

Chinese Academy of Medical Sciences \& Peking Union Medical College Hospital Jinsong Lei

Chinese Academy of Medical Sciences \& Peking Union Medical College Hospital

\section{Leyin Xu}

Chinese Academy of Medical Sciences \& Peking Union Medical College Hospital

\section{Xin Zhang}

Chinese Academy of Medical Sciences \& Peking Union Medical College Hospital

Xiu Liu

Chinese Academy of Medical Sciences \& Peking Union Medical College Hospital Zhichao Lai

Chinese Academy of Medical Sciences \& Peking Union Medical College Hospital Bao Liu ( $\sim$ liubao72@aliyun.com )

Peking Union Medical College Hospital https://orcid.org/0000-0001-6359-959X

\section{Research article}

Keywords: infrarenal aortic occlusion, endovascular treatment, bypass surgery

Posted Date: October 2nd, 2019

DOI: https://doi.org/10.21203/rs.2.11015/v2 
License: (c) (i) This work is licensed under a Creative Commons Attribution 4.0 International License. Read Full License 


\section{Abstract}

Background: Infrarenal aortic occlusion (IAO) is a complete occlusion of infrarenal aorta, which is the most complicated and severe subclass of aortoiliac occlusive disease (AIOD). The first-line treatment for IAO is bypass surgery due to its favorable patency rate. As endovascular technique advances, several retrospective studies have reported their endovascular experience for IAO. However, whether endovascular treatment(EVT) is comparable to bypass surgery for IAO required further study. This research was to study the safety and efficacy of EVT for IAO compared to bypass surgery. Methods: From 2003 to 2017, 44 consecutive patients with IAO was treated with $\operatorname{EVT}(n=16)$ or aortofemoral bypass surgery $(n=28)$. The therapeutic strategy depended on patient's will and state of health. Demographics, comorbidity, mortality, complication rate, and symptom-free survival proportion was compared by T-tests or chi-square test, to illustrate the safety and efficacy of EVT and bypass surgery. Results:The demographics and pre-operative Rutherford classification equally distributed in the two groups $(P>0.05)$. As for technical success, comorbidities, mortality, complication rate, and Rutherford classification after procedures, clinical success, no significant difference was observed $(P>0.05)$. The median post-procedure hospital stay was 4 days in the EVT group and was significantly shorter than that of the bypass, which was 11 days $(P<0.05)$. As for short-term and long-term results, the 1-year, 3-year, and 5-year the cumulative symptom-free survival rates were $85.7 \%, 85.7 \%$ and $85.7 \%$ in the EVT group, $100 \%, 94.1 \%$ and $80.7 \%$ in the aortofemoral bypass group.There was no significant difference insymptom-free survival ratebetween the two groups according to log-rank test $(P=0.92)$ Conclusions: The safety and efficacy of EVT were comparable to that of anatomic bypass surgery for IAO. EVT could be a feasible option for IAO.

\section{Introduction}

Infrarenal aortic occlusion(IAO) is a complete occlusion of infrarenal aorta, which classifies as TransAtlantic Inter-Society Consensus(TASC II) Type D lesions(1). It is the most complicated and severe subclass of aortoiliac occlusive disease(AIOD). Symptoms of IAO include intermittent claudication, loss or weakening of femoral artery beats, and erectile dysfunction in male patients(2). The incidence of IAO is low(prevalence, $0.15 \%)(3)$, accounting for only $3 \% \sim 8.5 \%$ of AIOD(4). The TASC II guidelines recommended bypass surgery for IAO due to its favorable primary patency rates(1). As endovascular technique advances, several groups have reported case series about their endovascular experience for IAO(5-9). In 2017, the European Society for Vascular Surgery(ESVS) proposed that endovascular treatment(EVT) could be first-line therapy for severe cases of AIOD who could not tolerate surgery. For patients who could tolerate surgery, aortofemoral bypass surgery was recommended, and EVT was an alternative(10). However, it remains unknown whether the safety and efficacy of EVT for IAO are comparable to that of bypass surgery. This study reviewed a total of 44 IAO patients treated surgically at PUMCH center retrospectively, and the patients were divided into EVT group and aortofemoral bypass group, to compare the safety and efficacy of EVT versus bypass surgery for IAO.

\section{Methods}




\section{Inclusion and exclusion criteria}

\section{Inclusion criteria:}

1.This study included all the consecutive patients with chronic(duration $>6$ months) and atherosclerotic total infrarenal aortic occlusion, who were treated surgically as inpatient at Peking Union Medical College Hospital, from January 2003 to December 2017. For all the patients, the occlusion ranged from infrarenal aortic artery to bilateral iliac artery, and common femoral artery was not involved. The diagnosis was confirmed by computed tomography for all patients, and angiography if necessary. Our center performed the first EVT for IAO in 2011 (for bypass group, 8 out of 28 patients received the surgery before 2011). After EVT has been employed for IAO, the patient's will was decisive based on all-round explanation about pros and cons of different therapeutic strategies. The Institutional Review Board of Peking Union Medical College Hospital approved this study, and all patients had signed informed content.

2.The patient was treated with EVT or aortofemoral bypass surgery.

Exclusion criteria『Patients with the inflammatory vascular disease were excluded from the study.

\section{Treatment strategy}

EVT: All endovascular cases were performed under local anesthesia by a surgical team. The left brachial artery was punctured, a catheter combined with a guidewire passed through the aortic arch and the descending aorta to the abdominal aorta. Then the surgeon replaced the catheter with a $6 / 7 \mathrm{~F} 90 \mathrm{~cm}$ long sheath. The catheter, along with the 0.035 or 0.018 guidewire, was delivered through the occlusive segments to the true lumen of the femoral artery. The femoral arteries were punctured, a short sheath implanted, and the working guidewire reestablished through the sheath side by side. The surgeon predilated the occlusion segments of the abdominal aorta and the iliac artery using a balloon (for example, Reekross 4-120 mm), and implanted two stents(for example, Medtronic $8 * 100,8 * 120 \mathrm{~mm}$ ) along with the guidewire by kissing technique. If the lesion was not covered completely, a stent of suitable length implanted jointly. Then we expanded the occlusive part again inside the stents with a balloon(for example, ADMIRAL $6 * 80 \mathrm{~mm}$ or longer). The detailed information was shown in Supplementary 1 Table 1 ; the specific stent type depended on the surgeon's preference and the lesion. After that, the surgeon performed angiography to confirm blood flow and apposition of the stents.

Aortofemoral bypass: Surgeries were performed under general anesthesia using a transperitoneal midline incision and longitudinal inguinal incision to expose the abdominal aorta and the femoral arteries, respectively. After heparinization, the surgeon clamped the abdominal aorta at a suitable level according to the upper edge of the occlusion. If renal arteries were involved, then they needed to be clamped as well. The surgeon cut the abdominal aorta, $2 \mathrm{~cm}$ below the level of the renal artery and then stripped out the thrombus. A bifurcated artificial graft (GORE-TEX $16-8 \mathrm{~mm}$ ) was employed retroperitoneally, and an endto-side anastomosis at the aorta and femoral artery were performed with Prolene3-0 or 5-0,respectively.

\section{Data analyses}


Quantitative data are described as mean and compared by independent t-tests. Categorical data are summarized as frequency and compared by chi-square tests. Ordinal categorical data are compared by the rank-sum test. The curve of the accumulative probability of survival was determined by the KaplanMeier method. P values were all two-sided, and statistical significance was defined as P区.050. All statistical analyses were performed by using GraphPad Prism (5.0v; GraphPad Software Inc).

\section{Follow up}

All cases were followed up at 0 months, 6 months, and 12 months postoperatively. Duration of follow-up was the period from the surgery to the last outpatient visit. Technical success was defined as revascularization of the occluded artery, and clinical success was defined as Rutherford classification returned to class 0 . The surgeon reevaluated the symptoms, artery pulses of the lower limb, and anklebrachial index (ABI). Symptom-free was defined as the no aggregation of claudication, no loss of

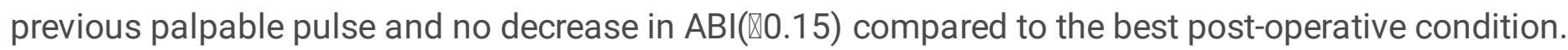

\section{Results}

\section{Demographics and preoperative comorbidities}

Between January 2003 and December 2017, 44 IAO patients visited Peking Union Medical College Hospital. 16 patients received EVT, 28 patients received aortofemoral bypass. The mean age $61.9 \mathrm{y}$ for the EVT group, 56.7 for the bypass group) and male proportion(93.8\% for the EVT group, $96.4 \%$ for the bypass group) of the two groups were similar(P区.050 each). Cardiovascular risk factors, including hypertension, diabetes mellitus, coronary artery disease hyperlipidemia, smoking, and homocysteinemia were equally prevalent between the two groups( $\mathrm{P} \otimes .050$ each). As for other comorbidities, 1 patient had renal insufficiency, and 10 patients had cerebral infarction in total, both of them were equally common between the two groups( $P \mathbb{0} .050$ each)(Table 1).

\section{Preoperative Rutherford classification and physical examination}

Most patients had severe claudication. According to the Rutherford classification, $11.4 \%$ of patients $(n=5)$ were class II, $61.4 \%(n=27)$ were class III, $18.2 \%(n=8)$ were class IV, $6.8 \%(n=3)$ were class V. Preoperative physical examination showed that most patients had diminished arterial pulse and a significant decrease in $A B I$ (left side: total 0.24 ,EVT 0.18 , bypass 0.33 ; right side: total 0.29 , EVT 0.34 , bypass 0.26 )(Table 2 ). The two groups were matched in Rutherford classification(P区.050) and ABI (left side, $p=0.084$; right side, $\mathrm{p}=0.42)($ Table 2$)$

\section{Perioperative period}

Revascularization was all achieved in the aortofemoral bypass group $(100 \%, \mathrm{n}=28)$. In the EVT group, there were 2 cases of technical failure, and the technical success rate was $87.5 \%(14 / 16)$. One case was that the guidewire could not pass through the occluded segment to establish a working route. Considering the patient's advanced age(>80 years old) and cardiac dysfunction(ejection fraction 39\%), 
the surgeon did not perform further treatment. The other case was that the patient underwent catheterdirected thrombolysis before EVT, and then acute renal infarction occurred(Cr $146 \mathrm{mmol} / \mathrm{L})$. The patient was discharged with conservative treatment. 3 months later, this patient received non-anatomic bypass surgery(axillo-bifemoral bypass). The postoperative Rutherford classification was 0 with a follow up of 34 months. Both of the 2 failed EVT cases were not accounted into the calculation of postoperative Rutherford classification. However, due to the small sample size, there was no statistical difference in the technical success rate between the two groups $(P>0.05)$. The 2 severe complications in the EVT group were acute kidney failure and retroperitoneal hematoma, respectively; the 7 severe complications of the aortofemoral bypass group were severe atrial fibrillation, severe pneumonia, infection of the graft, anaphylactic shock due to anesthesia, severe acute coronary syndrome, acute anterior myocardial infarction combined with pneumonia, massive pleural effusion with postoperative intestinal obstruction, respectively. The proportion of serious complications in EVT the group was less than that of the bypass group (12.5\%(2/16) in the EVT group, $25 \%(7 / 28)$ in the aortofemoral bypass group), but there was no statistical difference due to the small sample size(Table 3.). A representative example of the EVT procedure was shown in figure 1. The duration of hospital stays was 4 days for the EVT group, 11 days for the bypass group(Table 3.). There was a significant difference in hospital stay between the EVT group and the bypass groups $(P<0.05)$.

\section{Follow-up}

40 out of 44 patients had an effective follow-up after surgery. The median follow-up period was 50.9 months. 31 out of the 40 patients' symptoms disappeared after the operation and reached an asymptomatic state (Table 3). The 1-year, 3-year, and 5-year cumulative symptom-free survival rates were $85.7 \%, 85.7 \%$ and $85.7 \%$ in the EVT group, $100 \%, 94.1 \%$ and $80.7 \%$ in the aortofemoral bypass group. There was no significant difference in symptom-free survival rate between the two groups according to the log-rank test $(P=0.92)$ (figure 1$)$. The evaluation of primary and secondary patency was not conducted because a majority of asymptomatic patients had not performed any imaging examinations after surgery.

\section{Discussion}

Aortofemoral bypass has been performed to treat IAO since the 1960s; it is still the first-line treatment of IAO nowadays. A recent meta-analysis showed that the perioperative mortality rate is $0 \%-1.9 \%(11)$.

Surgical techniques have developed to reduce ischemic damage of kidney in proximal IAO $(11,12)$. Recent research shows that its 5 -year patency rate is $88.6 \%(4)$. In our center, 20 of 24 patients returned to the asymptomatic state, and the accumulative probability of survival was $80.7 \%$ for 5 years. Also, there was no perioperative death in our study, but $25 \%$ (7/28) patients had life-threatening complications.

Suggesting that comprehensive evaluation is necessary to select patients who can tolerate the bypass surgery. 
Laparoscopic aorto-bifemoral bypass surgery, compared with conventional open surgery, its complications are similar or fewer[10,11], the postoperative hospital stay is shorter[10,12], and expenditure is lesser[13]. Retrospective cohort analysis showed that the 5-year primary and secondary patency rates were as high as $83 \%$ and $97 \%[14]$. But the number of reported cases was few, bulk data are needed for further support. Because of the long learning curve of laparoscopic bypass[15] and the advantage of fewer injuries of EVT, there are not many centers that perform laparoscopic bypass surgery. And our center didn't perform laparoscopic aorto-bifemoral bypass surgery.

With the rapid development of EVT, the guideline has recommended EVT as the preferred treatment for short stenosis or short occlusion of the iliac artery segment (types A and B in the TASC grading)(1). Unlike type $A$ and $B, I A O$ (type $D$ ) involves the problems about the establishment of guidewire pathways. If a recanalization cannot be established, EVT cannot be performed. In order to increase the success rate of establishing working pathways, "double-barrel" self-expanding stents(13), catheter-directed thrombolysis and even hybrid surgery can be used. As in our EVT group ( $n=16), 4$ patients did catheter-directed thrombolysis, and 2 patient performed femoral endarterectomy as an adjunctive treatment(Supplementary 1 Table 1). For extensive stenosis or occlusion of the aortoiliac artery (TASC grading, types $C$ and D), a recent meta-analysis shows that EVT also has a technical success rate of $90 \%$ and a one-year primary patency rate of nearly $90 \%(14)$. Cases of EVT for IAO have been reported in the early 1990 s $(15,16)$, and more case series study has appeared in recent years $(6,8,9,17,18)$. A cases series including 49 IAO patients treated with EVT reported the primary patency rate was $88.4 \%$ at 1 year and $80.1 \%$ at 3 years(6). Another case series including 31 IAO patients reported the primary patency rates were $85 \%$ and $66 \%$ at 1 and 3 years, respectively(5). As more and more data have shown satisfied outcomes of EVT for treating IAO, whether EVT is comparable to the first-line aortofemoral bypass surgery required to be answered. And this study proved that EVT is as safe and effective as compared to aortofemoral bypass surgery.

Our study had several limitations. It was a retrospective study; the patients were divided nonrandomly into EVT group and bypass group. Moreover, the small sample size of our study also limited statistical comparisons.

\section{Conclusions}

We reported a retrospective study of EVT or bypass treated IAO in our center. The demographics and preoperative Rutherford classifications were equally distributed in the two groups. Technical success rate, mortality rate, complications rate, and Rutherford classification after procedures, and 1-year, 3-year and 5year symptom-free survival proportion were compared between the 2 groups, and there was no significant difference between the 2 groups. Therefore, the safety and efficacy of EVT for IAO is comparable to that of bypass surgery, and EVT could be a feasible choice for the treatment of IAO patients.

\section{Abbreviations}


IAO Infrarenal aortic occlusion

AIOD aortoiliac occlusive disease

EVT endovascular treatment

\section{Declarations}

Ethics approval and consent to participate: The Institutional Review Board of Peking Union Medical College Hospital approved this study and written informed consent was obtained from all the participants.

Consent for publication: Not applicable.

Availability of data and material: The datasets generated and analyzed during the current study are all included in the article or supplementary materials.

Competing interests: The authors declare that they have no competing interests.

Funding: This study was supported by Beijing Municipal Science and Technology Commission: No. Z161100000516093; Clinical Trials. gov ID: NCT03248323.

Authors' contributions: All authors contributed significantly to this work; all authors revised the manuscript and gave final approval of the manuscript to be submitted. JJW, CYQ, and BL designed the study, CYQ, JS, KL, LJF, JSL, LYX, XZ, XL, ZCL analyzed and interpreted the data, JJW and BL drafted the article.

Acknowledgements: Not applicable.

\section{References}

1. Norgren L, Hiatt WR, Dormandy JA, Nehler MR, Harris KA, Fowkes FG, et al. Inter-Society Consensus for the Management of Peripheral Arterial Disease (TASC II). J Vasc Surg. 2007;45 Suppl S:S5-67.

2. Ioncioaia B, Bud IT, Muresan M. Rene leriche and the development of 20(th) century surgery. Clujul Med. 2016;89(1):176-80.

3. Starer F, Sutton D. Aortic thrombosis. Br Med J. 1958;1(5082):1255-63.

4. Marrocco-Trischitta MM, Bertoglio L, Tshomba Y, Kahlberg A, Marone EM, Chiesa R. The best treatment of juxtarenal aortic occlusion is and will be open surgery. J Cardiovasc Surg (Torino). 2012;53(3):307-12.

5. Moise MA, Alvarez-Tostado JA, Clair DG, Greenberg RK, Lyden SP, Srivastava SD, et al. Endovascular management of chronic infrarenal aortic occlusion. J Endovasc Ther. 2009;16(1):84-92. 
6. Kim TH, Ko YG, Kim U, Kim JS, Choi D, Hong MK, et al. Outcomes of endovascular treatment of chronic total occlusion of the infrarenal aorta. J Vasc Surg. 2011;53(6):1542-9.

7. Yuan L, Bao J, Zhao Z, Feng X, Lu Q, Jing Z. Endovascular therapy for long-segment atherosclerotic aortoiliac occlusion. J Vasc Surg. 2014;59(3):663-8.

8. Mangialardi N, Ronchey S, Serrao E, Fazzini S, Alberti V, Orrico M, et al. Endovascular management of total juxtarenal aortic occlusive disease in high-risk patients: technical considerations and clinical outcome. J Cardiovasc Surg (Torino). 2017;58(3):422-30.

9. Morisaki K, Yamaoka T, Iwasa K, Ohmine T. Outcomes of Endovascular Therapy for Infrarenal Aortic Occlusion of TASC II D Classification. Ann Vasc Surg. 2017;43:203-9.

10. Aboyans V, Ricco JB, Bartelink MEL, Bjorck M, Brodmann M, Cohnert T, et al. 2017 ESC Guidelines on the Diagnosis and Treatment of Peripheral Arterial Diseases, in collaboration with the European Society for Vascular Surgery (ESVS). Eur Heart J. 2018;39(9):763-816.

11. Yang SS, Kim YW, Park YJ, Kim DI, Woo SY, Huh S, et al. Results of Open Surgical Repair of Chronic Juxtarenal Aortic Occlusion. Vasc Specialist Int. 2014;30(3):81-6.

12. Pearce FB, Jr., Yang S, Shi R, Tan TW, Zhang WW. Circumferential Aortic Endarterectomy Followed with Immediate Infrarenal Clamping Obviates Suprarenal Clamping for Juxtarenal Aortoiliac Occlusion. Ann Vasc Surg. 2016;34:48-54.

13. Varcoe RL, Nammuni I, Lennox AF, Walsh WR. Endovascular reconstruction of the occluded aortoiliac segment using "double-barrel" self-expanding stents and selective use of the Outback LTD catheter. J Endovasc Ther. 2011;18(1):25-31.

14. Ye W, Liu CW, Ricco JB, Mani K, Zeng R, Jiang J. Early and late outcomes of percutaneous treatment of TransAtlantic Inter-Society Consensus class $C$ and D aorto-iliac lesions. J Vasc Surg. 2011;53(6):1728-37.

15. Diethrich EB. Endovascular techniques for abdominal aortic occlusions. Int Angiol. 1993;12(3):27080.

16. Pilger E, Decrinis M, Stark G, Koch G, Obernosterer A, Tischler R, et al. Thrombolytic treatment and balloon angioplasty in chronic occlusion of the aortic bifurcation. Ann Intern Med. 1994;120(1):40-4.

17. Kasemi H, Marino M, Dionisi CP, Di Angelo CL, Fadda GF. Seven-Year Approach Evolution of the Aortoiliac Occlusive Disease Endovascular Treatment. Ann Vasc Surg. 2016;30:277-85.

18. Schmalstieg J, Zeller T, Tubler T, Sixt S, Schwencke C, Sandstede J, et al. Long term data of endovascularly treated patients with severe and complex aortoiliac occlusive disease. J Cardiovasc Surg (Torino). 2012;53(3):291-300.

\section{Tables}

Table 1. Demographic data and preoperative comorbidities 


\begin{tabular}{|c|c|c|c|c|}
\hline & Total & EVT & Bypass & $P$ value \\
\hline Number & 55 & 16 & 28 & \\
\hline Mean age $\bigotimes$ range $\rrbracket$ & $58.4 \rrbracket 45-82 \rrbracket$ & $61.9 \llbracket 45-82 \square$ & $56.7 \rrbracket 45-78 \rrbracket$ & $>0.05(0.059)$ \\
\hline Male & $95.5 \rrbracket 42 / 44 \rrbracket$ & $93.8 \otimes 15 / 16 \rrbracket$ & $96.4 \llbracket 27 / 28 \rrbracket$ & $>0.05(0.68)$ \\
\hline Hypertension & $50.0 \rrbracket 22 / 44 \rrbracket$ & $68.8 \otimes 11 / 16 \rrbracket$ & $39.3 \otimes 11 / 28 \rrbracket$ & $>0.05(0.060)$ \\
\hline Diabetes mellitus & $11.4 \llbracket 5 / 44 \rrbracket$ & $6.3 \otimes 1 / 16 \rrbracket$ & $14.3 \rrbracket 4 / 28 \rrbracket$ & $>0.05(0.42)$ \\
\hline Coronary artery disease & $15.9 \otimes 7 / 44 \rrbracket$ & $25.0 \rrbracket 4 / 16 \rrbracket$ & $10.7 \rrbracket 3 / 28 \rrbracket$ & $>0.05(0.21)$ \\
\hline Renal insufficiency & $2.3 \otimes 1 / 44 \rrbracket$ & $6.3 \otimes 1 / 16 \rrbracket$ & $0 \otimes 0 / 28 \rrbracket$ & $>0.05(0.18)$ \\
\hline Cerebral infarction & 22.7凶10/44】 & 18.8ه3/16ه & $25.0 \otimes 7 / 28 \rrbracket$ & $>0.05(0.63)$ \\
\hline Hyperlipidemia & $25.0 \otimes 11 / 44 \rrbracket$ & $31.2 \varangle 5 / 16 \rrbracket$ & $21.4 \rrbracket 6 / 28 \rrbracket$ & $>0.05(0.47)$ \\
\hline Smoking & 88.6冈39/44区 & $93.8 \otimes 15 / 16 \rrbracket$ & $85.7 \rrbracket 24 / 28 \rrbracket$ & $>0.05(0.42)$ \\
\hline Smoking index* & 861.5 & 826.7 & 883.3 & $>0.05(0.75)$ \\
\hline Homocysteinemia & $32.3 \otimes 10 / 31 \rrbracket$ & $23.1 \rrbracket 3 / 13 \rrbracket$ & $38.9 \otimes 7 / 18 \rrbracket$ & $>0.05(0.20)$ \\
\hline
\end{tabular}

*Smoking index=years of smoking $\times$ number of cigarettes per day, the unit is package year.

Table 2. Rutherford classification and physical examination 


\begin{tabular}{lccc}
\hline \multicolumn{1}{c}{ Total } & EVT & Bypass & P value \\
\hline Rutherford classification & & & $0.05(1.00)$ \\
I & 1 & 1 & 4 \\
\hline II & 5 & 1 & 17 \\
\hline III & 27 & 10 & 5 \\
\hline IV & 8 & 3 & 2 \\
\hline V & 3 & 1 & 0
\end{tabular}

Artery pulse

\begin{tabular}{|c|c|c|c|c|}
\hline left femoral & $14.0 \otimes 6 / 43 \rrbracket$ & $18.8 \rrbracket 3 / 16 \rrbracket$ & $11.1 \rrbracket 3 / 27 \rrbracket$ & $>0.05(0.48)$ \\
\hline left popliteal & $7.1 \otimes 3 / 42 \rrbracket$ & $6.7 \rrbracket 1 / 15 \rrbracket$ & $7.4 \llbracket 2 / 27 \rrbracket$ & $>0.05(0.93)$ \\
\hline left posterior tibial & $0 \rrbracket 0 / 43 \rrbracket$ & $0 \rrbracket 0 / 16 \rrbracket$ & $0 \rrbracket 0 / 27 \rrbracket$ & $>0.05(1.00)$ \\
\hline left dorsal & $4.5 \rrbracket 2 / 44 \rrbracket$ & $6.3 \otimes 1 / 16 \rrbracket$ & $3.6 \rrbracket 1 / 28 \rrbracket$ & $>0.05(0.68)$ \\
\hline right femoral & $11.4 \rrbracket 5 / 44 \rrbracket$ & $12.5 \rrbracket 2 / 16 \rrbracket$ & $10.7 \rrbracket 3 / 28 \rrbracket$ & $>0.05(0.86)$ \\
\hline right popliteal & $4.7 \rrbracket 2 / 42 \rrbracket$ & $0 \rrbracket 0 / 15 \rrbracket$ & $7.4 \llbracket 2 / 27 \rrbracket$ & $>0.05(0.28)$ \\
\hline right posterior tibial & $2.3 \rrbracket 1 / 43 \rrbracket$ & $0 \otimes 0 / 16 \rrbracket$ & $3.7 \rrbracket 1 / 27 \rrbracket$ & $>0.05(0.44)$ \\
\hline right dorsal & $6.8 \rrbracket 3 / 44 \rrbracket$ & $12.5 \rrbracket 2 / 16 \rrbracket$ & $3.6 \rrbracket 1 / 28 \rrbracket$ & $>0.05(0.26)$ \\
\hline left $A B I$ & 0.24 & 0.33 & 0.18 & $>0.05(0.084)$ \\
\hline right $A B I$ & 0.29 & 0.34 & 0.26 & $>0.05(0.42)$ \\
\hline
\end{tabular}

Table 3. Peri-operative period and follow-up 


\begin{tabular}{|c|c|c|c|c|}
\hline & Total & EVT & Bypass & $P$ value \\
\hline Technical success rate & 42 & $87.5 \rrbracket 14 / 16 \rrbracket$ & $100 \rrbracket 28 / 28 \rrbracket$ & $>0.05(12.7)$ \\
\hline NO. of complications & 22 & 5 & 13 & $>0.05(0.32)$ \\
\hline NO. of severe complications* & 9 & 2 & 7 & $>0.05(0.32)$ \\
\hline Length of stay (day) & $10.8(2-27)$ & $7.9(2-24)$ & $12.4(8-27)$ & $<0.05(0.005)$ \\
\hline NO. of effective follow-up & 40 & 16 & 24 & $<0.05(0.11)$ \\
\hline Follow-up (month) & 50.9 & 37.5 & 59.0 & $>0.05(0.057)$ \\
\hline \multicolumn{5}{|c|}{ Postoperative Rutherford classification (it was performed about 2 weeks after surgery) } \\
\hline asymptomatic & 31 & 11 & 20 & \\
\hline I & 1 & 0 & 1 & \\
\hline II & 1 & 0 & 0 & \\
\hline III & 3 & 2 & 1 & \\
\hline IV & 0 & 0 & 0 & \\
\hline $\mathrm{V}$ & 0 & 0 & 0 & \\
\hline VI & 0 & 0 & 0 & \\
\hline Clinical success rate & $70.5 \%$ & $68.8 \%$ & $71.4 \%$ & $>0.05(0.85)$ \\
\hline
\end{tabular}

Definition of severe complications: life-threatening bleeding, infection, or acute coronary disease.

*The 2 severe complications in the EVT group: acute kidney failure and retroperitoneal hematoma, respectively; the 7 severe complications of anatomic group: severe atrial fibrillation, severe pneumonia, infection of the graft, anaphylactic shock due to anesthesia, severe acute coronary syndrome, acute anterior myocardial infarction combined with pneumonia, massive pleural effusion with postoperative intestinal obstruction, respectively.

\section{Figures}




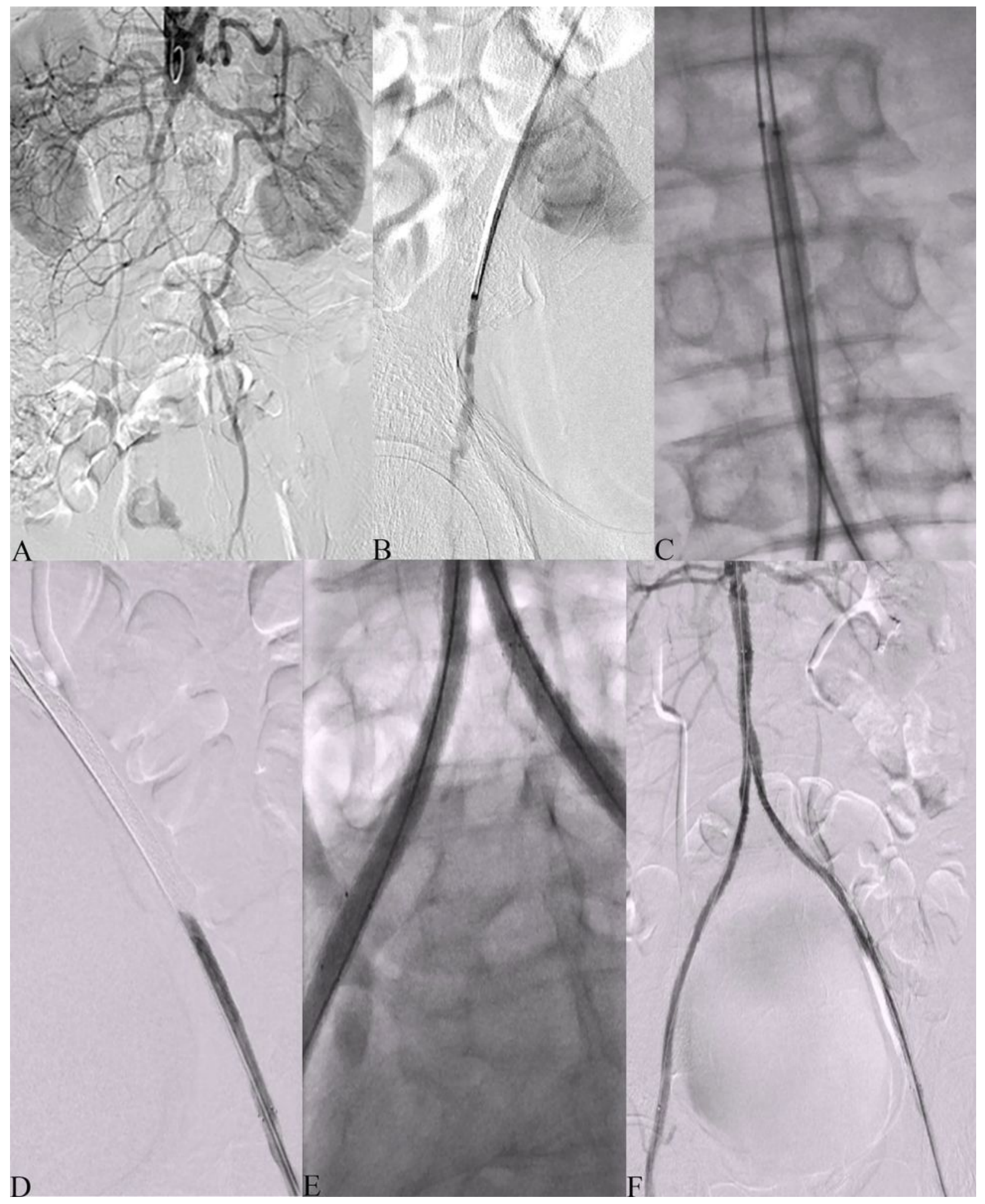

\section{Figure 1}

A representative example of EVT. A. An angiography showed collateral circulation, confirming the diagnosis of IAO. B. A catheter with guidewire fitted through the occlusion segment. C. Balloons predilated the occlusive segment of the aortoiliac artery with the kissing technique. D. A stent was released in one of the iliac branches, and the angiography showed a good distal outflow. E. After stents were 
released, a balloon expanded the aortoiliac artery after insides the stents. F. The angiogram shows that the blood flow within the stent is reasonable, and the flow rate is satisfactory.

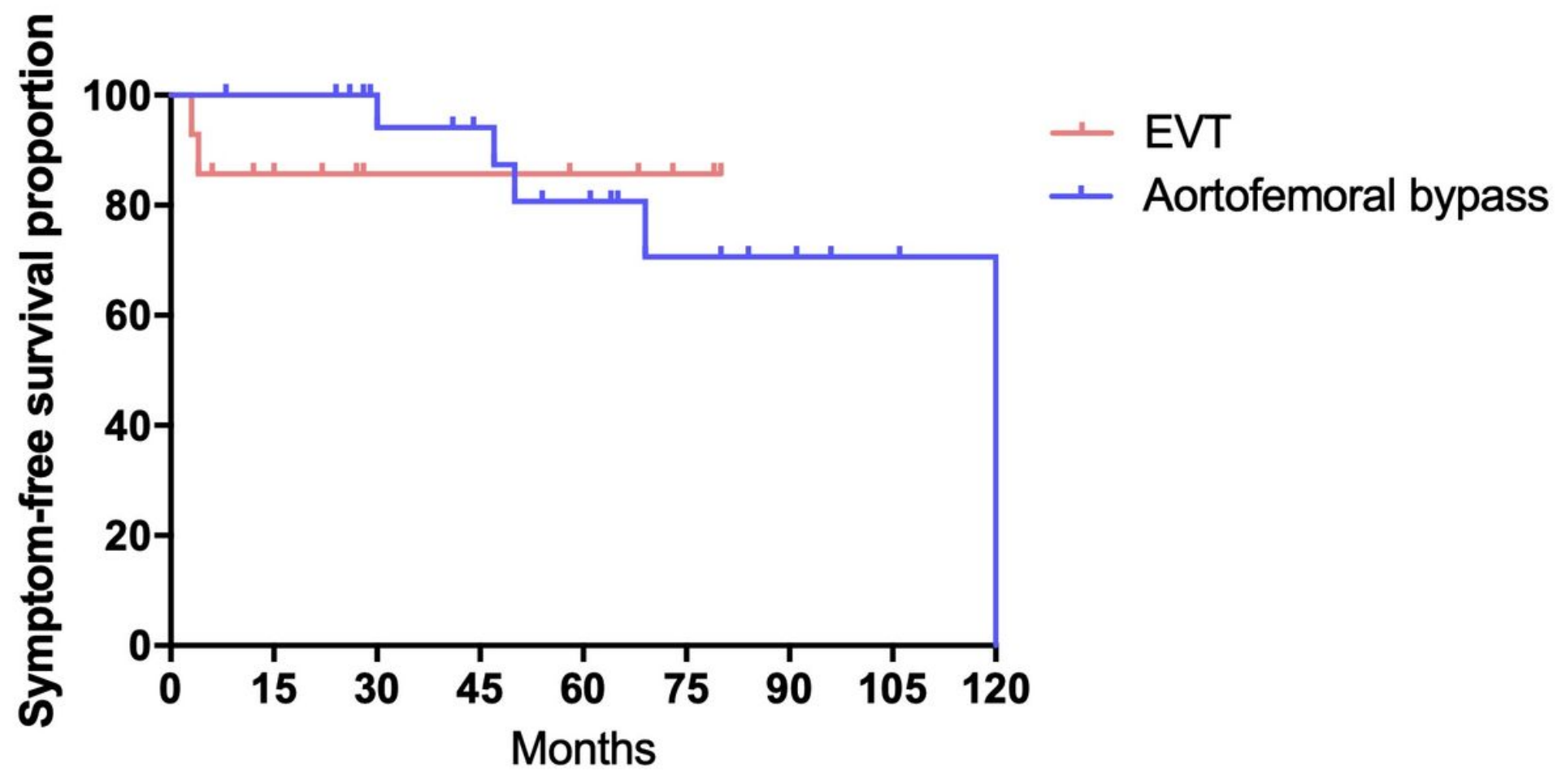

Figure 2

Asymptomatic survival curve in patients who were asymptomatic after procedures. (EVT group $n=11$, aortofemoral bypass group $n=20$ ).

\section{Supplementary Files}

This is a list of supplementary files associated with this preprint. Click to download.

- Supplemantary1revised.pdf 